\title{
PRINCIPAIS MANIFESTAÇÕES PATOLÓGICAS NA COBERTURA DE EDIFÍCIOS DO BAIRRO ASA SUL, BRASÍLIA-DF
}

\author{
CAVALCANTE, CAROLINNE LISBOA \\ Engenheira Civil \\ Centro Universitário do Distrito Federal - UDF \\ Distrito Federeal; Brasil \\ carolinnelisboacavalcante@ hotmail.com
}

\author{
CHATER, LATIF \\ Engenheiro Civil \\ JR \& Chater \\ Distrito Federal; Brasil \\ jrchater@gmail.com
}

\author{
SILVA, JULIANO RODRIGUES \\ Docente de Engenharia Civil \\ Universidade Estadual de Goiás \\ Goiás; Brasil \\ julianorodriguessilva@gmail.com
}

\author{
SCHMIDT, ANDREIA \\ Engenheira Civil \\ Universidade Fernando Pessoa \\ Porto, Portugal \\ eng.andreiaschmidt@gmail.com
}

\begin{abstract}
RESUMO
O trabalho apresenta as principais manifestações patológicas na cobertura de edifícios residenciais no bairro Asa Sul, que é um bairro da região administrativa de Brasília, no Distrito Federal, sendo uma área tombada pela UNESCO (Organização das Nações Unidas para a Educação, a Ciência e a Cultura). Na cobertura das edificações, foram identificados problemas como desgaste no sistema de impermeabilização, sistema importante, visto que as lajes de cobertura sofrem exposição direta de intempéries como sol e chuva., falta de calafetação de telhas, telhas desgastadas pelo tempo e buzinotes (drenos) obstruídos. Todas as informações referentes às manifestações patológicas citadas foram obtidas através de vistorias nas próprias edificações. Dessa forma, a inspeção predial foi baseada na vistoria visual da edificação, que tem como resultado a análise técnica do fato ou da condição relativa à utilização, mediante a verificação "in loco" de cada cobertura, no que tange a segurança e a manutenção predial, que foram pautadas de acordo com as diretrizes da Norma de Inspeção Predial do IBAPE (Instituto Brasileiro de Avaliações e Perícias de Engenharia) de 2012, no seu Nível de inspeção 01 e as especificações da Norma de Manutenção em Edificações - NBR 5674 (1999), da ABNT (Associação Brasileira de Normas Técnicas). De uma forma geral, pode-se dizer que os demais problemas surgem em virtude dos edifícios serem antigos, além de terem sofrido diversas intervenções ao longo de sua história, resultando em anomalias construtivas. Muitos de seus elementos construtivos se encontram, portanto, em situações suscetíveis à apresentação de manifestações patológicas, pois estão se deteriorando naturalmente.
\end{abstract}

Palavras-chave: cobertura, manifestações patológicas, edifícios, impermeabilização.

\section{ABSTRACT}

The paper presents the main pathological manifestations on the roof of residential buildings in the Asa Sul neighborhood, which is a district of the administrative region of Brasilia, in the Distrito Federal, being an area listed by UNESCO (United Nations Educational, Scientific and Cultural Organization). On the roof of the building, problems were identified as wear and tear on the waterproofing system, an important system, since the roof slabs suffer direct exposure of weather such as sun and rain, lack of caulking tiles, weathered tiles and obstructed horns. All information regarding the pathological manifestations mentioned was obtained through surveys in the buildings themselves. Thus, the building inspection was based on the visual inspection of the building, which results in the technical analysis of the fact or condition related to the use, through the on-site verification of each roof, regarding safety and building maintenance, which were guided according to the guidelines of the Building Inspection Standard of IBAPE (Brazilian Institute of Engineering Assessment and Expertise) of 2012, in its Inspection Level 01 and the specifications of Building Maintenance Standard - NBR 5674 (1999). , from ABNT (Brazilian Association of Technical Standards). In general, it can be said that the other problems arise because the buildings are old, and have undergone various interventions throughout their history, resulting in constructive anomalies. Many of its constructive elements are therefore in situations susceptible to the presentation of pathological manifestations, as they are naturally deteriorating. 


\section{INTRODUÇÃO}

Segundo Souza e Ripper (1998), é chamada de patologia das estruturas, a área da engenharia civil que se ocupa do estudo das origens, formas de manifestação, consequências e mecanismos de ocorrência das falhas e dos sistemas de degradação das estruturas. É uma área que vai além da identificação e conhecimento das anomalias, se relaciona, também, à concepção e ao projeto das estruturas, e se estende, até mesmo, à formação do engenheiro civil. Para Gomide, Fagundes Neto e Gullo (2009), podem ser consideradas como sendo o estudo das modificações das condições físicas e/ou funcionais causadas por anomalias ou falhas nas edificações.

Tendo em vista, que mesmo estruturas bem projetadas e construídas apresentam sintomas patológicos devido ao desgaste natural da estrutura, as análises patólógicas são indispensáveis para assegurar um desempenho satisfatório ao longo da vida útil da construção. A fim de se obter sucesso, no que diz respeito, às soluções para os problemas patológicos diagnosticados é necessário que o diagnóstico tenha sido bem feito, que se conheça muito bem as vantagens e desvantagens de cada material, e para cada situação particular a melhor alternativa de solução.

Com o objetivo de minimizar o surgimento de patologias construtivas e auxiliar os profissionais desse setor, foram craidas normas técnicas sobre desempenho das edificações, manutenções, perícias e inspeções, com diretrizes e recomendações que devem ser seguidas com o propósito de manter o desempenho esperado das edificações. No Brasil, devido ao crescente número de incidentes envolvendo os estados de conservação das estruturas, notou-se a necessidade de melhorar as perícias técnicas, os laudos técnicos de inspeção predial e de conscientizar a população sobre a importância desse serviço para manter a segurança, o uso e ocupação dessas edificações.

As coberturas são sistemas que compõe o topo das edificações e, assim como qualquer um deles, está passível de sofrer com patologias e intempéries como sol e chuva, considerando que sua integridade interfere no desempenho de outros elementos que fazem parte da construção, analisar as manifestações patológicas nesse elemento é fundamental.

Dessa forma, esta artigo tem a intenção de analisar a conservação e vida útil na cobertura de edifícios residenciais no bairro Asa Sul, Brasília-DF, bem como, a identificação de anomalias e de falhas que se apresentam de forma aparente. Para cada manifestação patológica apontada nas coberturas foram apresentados registros fotográficos, assim como as sugestões para a solução dessas referidas patologias.

O sistema de cobertura é um dos sistemas que compõe as edificações e, assim como qualquer um deles, está passível de sofrer com as patologias, e considerando que sua integridade é de suma importância para o desempenho de outros elementos que fazem parte da construção, analisar as manifestações patológicas que ali surgem é de grande importância.

\section{METODOLOGIA}

As inspeções prediais foram baseadas na vistoria visual das edificações, que tem como resultado a análise técnica do fato ou da condição relativa à utilização, mediante a verificação "in loco" de cada sistema construtivo, no que tange a segurança e a manutenção predial, que foram pautadas de acordo com as diretrizes da Norma de Inspeção Predial do IBAPE (Instituto Brasileiro de Avaliações e Perícias de Engenharia) - 2012, no seu Nível de Inspeção 01 e as especificações da Norma de Manutenção em Edificações - NBR 5674 (1999), da ABNT.

A inspeção procede ao diagnóstico das anomalias construtivas e falhas de manutenção que interferem e prejudicam o estado de utilização do prédio e suas instalações, tendo como objetivo verificar os aspectos de desempenho, vida útil, utilização e segurança que tenham interface direta com os usuários. As normas de inspeção do IBAPE, estabelece três níveis quanto a complexidade e elaboração de laudo, consideradas as características técnicas da edificação, manutenção e operação existentes e necessidade de formação de equipe multidisciplinar para execução dos trabalhos:

Nível 1: a inspeção deve ser elaborada por profissional habilitado em uma especialidade, considerando-se uma edificação com baixa complexidade técnica, de manutenção e operação de seus elementos e sistemas construtivos.

Nível 2: a inspeção deve ser elaborada por profissional habilitado em uma ou mais especialidades, considerando-se uma edificação com média complexidade técnica, de manutenção e operação de seus elementos e sistemas construtivos;

Nível 3: é elaborada por profissionais habilitados e de mais de uma especialidade, nesse caso, o trabalho pode ser intitulado como auditoria técnica, considerando seu alto grau de complexidade técnica, de manutenção e operação. 
A inspeção realizada, para fins deste trabalho, é classificada como "Inspeção de Nível 01", representada por análise expedita dos fatos e sistemas construtivos vistoriados, com a identificação de suas anomalias e de falhas que se apresentam de forma aparente.

Caracteriza-se pela verificação isolada ou combinada das condições técnicas de uso e de manutenção do sistema da edificação, de acordo com a Norma de Inspeção Predial do IBAPE (2012), respeitado o nível de inspeção adotado, com a classificação das deficiências encontradas quanto ao grau de risco que representa em relação à segurança dos usuários, à habitabilidade e à conservação do patrimônio edificado.

Conforme a referida Norma de Inspeção Predial do IBAPE (2012), as anomalias e falhas são classificadas em três diferentes graus de recuperação (mínimo, regular e crítico), considerando o impacto do risco oferecido aos usuários, ao meio ambiente e ao patrimônio:

Grau de risco mínimo $(\mathbf{M})$ - Risco de causar pequenos prejuízos à estética ou atividade programável e planejada, sem incidência ou sem a probabilidade de ocorrência dos riscos críticos e regulares, além de baixo ou nenhum comprometimento do valor imobiliário a edificação.

Grau de risco regular (R) - Risco de provocar a perda parcial de desempenho e funcionalidade da edificação sem prejuízo à operação direta de sistemas, e deterioração precoce do edifício.

Grau de risco crítico $(\mathbf{C})$ - Risco de provocar danos contra a saúde e segurança das pessoas e do meio ambiente; perda excessiva de desempenho e funcionalidade causando possíveis paralisações; aumento excessivo de custo de manutenção e recuperação; comprometimento sensível de vida útil da edificação.

\section{MANIFESTAÇÕES PATOLÓGICAS}

\subsection{Desgaste no sistema de impermeabilização das coberturas}

De acordo com a NBR 9575 (2010), impermeabilização é definida como sendo um conjunto de operações e técnicas construtivas (serviços), compostos por uma ou mais camadas, que tem por finalidade proteger as construções contra a ação prejudicial dos fluidos, dos vapores e da umidade. O sistema de impermeabilização é importante, pois evita a deterioração da estrutura e minimiza os problemas de sáude relativos a umidade.

Manifestações patológicas: O problema mais comum, encontrado nas coberturas das edificações vistoriadas foi o desgaste no sistema de impermeabilização. Foram identificados alguns pontos de falha do revestimento aluminizado das mantas asfálticas de impermeabilização nas áreas sobre os reservatórios de água, o que pode já estar provocando infiltrações de água na estrutura do reservatório. Este material apresenta pequeno desgaste do revestimento aluminizado, conforme as Figuras 1 e 2.

As infiltrações podem provocar uma cadeia de eventos que contribuem para a degradação das estruturas de concreto armado favorecendo a corrosão das armaduras e consequente destacamento do concreto que o recobre. Além disso, ambientes internos dos apartamentos dos pavimento superiores podem perder condições de salubridade e habitabilidade por umidade excessiva, com geração de fungos que podem provocar problemas respiratórios.

Classificação do Problema: falha na manutenção e anomalia construtiva;

Causa: A camada de proteção aluminizada da manta sobre os reservatórios está extremamente exposta a radiação solar e outras intempéries. Este material, portanto, está sujeito a movimentos de expansão e retração constantes, com os ciclos climáticos diários, em que resfria e aquece. Recomenda-se a substituição do material desgastado pelo tempo de forma preventiva observando a declividade adequada da laje para os pontos de drenagem (mínimo $1 \%$ ) e a capacidade dos drenos de esgotar a água acumulada, com diâmetros suficientes para este fim. 

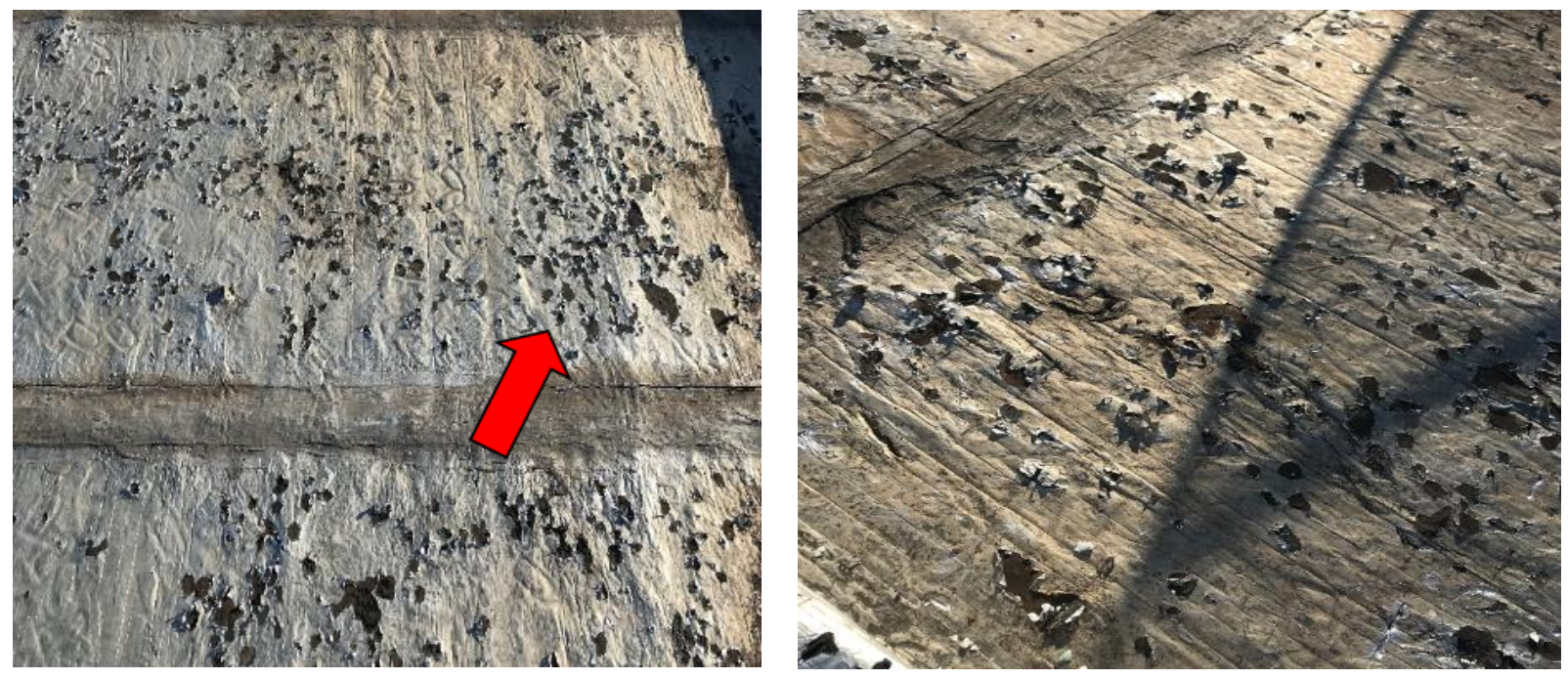

Figura 1: Impermeabilização das lajes dos reservatórios Figura 2: Impermeabilização das lajes dos reservatórios

Intervenção: Sugere-se verificar há quanto tempo que foram realizadas as últimas impermeabilizações e caso possuam idade superior a 10 anos (ou a idade de substituição indicada pelo fabricante), recomenda-se uma nova impermeabilização da cobertura, visando a prevenção de infiltrações futuras.

Caso se proceda à substituição da impermeabilização, sugere-se procedimento a seguir. Primeiramente, recomenda-se remover o material de impermeabilização existente. A NBR 9575 (2010) recomenda que seja feita a regularização das superfícies localizadas abaixo das mantas com argamassa desempenada de cimento e areia, com caimento mínimo de $1 \%$ em direção aos ralos. Arredondar ou chanfrar cantos vivos e arestas, de forma a permitir um ajustamento contínuo do sistema impermeabilizante, sem dobragem em ângulo, tubulações emergentes e ralos que deverão estar rigidamente fixados, a fim de garantir a perfeita execução dos arremates. A execução de arremates no ralo é, provavelmente, o detalhe mais importante do processo de impermeabilização. Tal execução é feita com aplicação de sucessivas demãos que adentram a abertura do piso, podendo ou não receber reforços de estruturantes têxteis e, quando aplicados à quente, devem ser dimensionados para suportar as temperaturas de aplicação. A já citada norma de impermeabilização, NBR 9575 (2010), refere que os coletores devem ter diâmetro que garanta a manutenção da seção nominal dos tubos prevista no projeto hidráulico após a execução da impermeabilização, sendo que o diâmetro nominal mínimo é 75 mm. A região em torno do ralo deve ser rebaixada para que se execute um reforço de impermeabilização, sendo que esta deve ficar bem aderida à face interna do ralo. Caso contrário, a água será succionada por capilaridade para baixo da camada impermeabilizante.

Quanto a aplicação da manta aluminizada, recomenda-se a limpeza do local, sem resíduos, restos de argamassa, madeiras, pontas de ferro, graxa, óleo e partículas soltas. Se necessário, lavar o local com hidrojateamento ou com escova de aço e água. Esperar secar. Para a aderência da manta em toda a área, incluindo rebaixos, aplicar uma demão de primer asfáltico. Aguardar a secagem do primer asfáltico, antes da colagem das mantas asfálticas aluminizadas. Com um maçarico de boca larga e gás GLP, aquecer o primer asfáltico e a parte inferior da manta asfáltica aluminizada até o plástico de proteção derreter. A chama do maçarico deve derreter superficialmente o filme de polietileno e o asfalto em toda a extensão da manta asfáltica aluminizada, deve-se evitar que a manta seja perfurada devido à intensidade e tempo prolongado de exposição à chama do maçarico. Sugere-se, nas paredes externas das casas de máquinas, caso as fissuras estejam realmente vedadas, a pintura com manta líquida com acabamento final com pintura (utilizar Vedapren Parede ou similar). Todos os serviços acima descritos devem ser executados por profissionais legalmente habilitados e especializados, com Anotação de Responsabilidade Técnica (ART) registrada junto ao CREA-DF.

Risco: M (Grau Mínimo). 


\section{СВРAT 2020 \\ concerss b a bus \\ DE 15 A 17 DE ABRIL | FORTALEZA - CE}

ISBN 978-65-86819-05-2

\subsection{Falta de calafetação e desgaste das telhas}

O envelhecimento do material que compõe as telhas, faz com que surjam frestas entre telhas; entre a telha e a cumeeira; e entre telhas e calhas, locais por onde água pode infiltrar. Esse problema surge, também, devido a calafetação feita de forma incorreta ou ineficiente.

Manifestações patológicas: Alguns pontos de contato entre telhas careciam de calafetação (Figuras 3 e 4), além disso, as telhas aparentam desgaste natural pelo tempo de uso, embora retenham sua capacidade de vedação. Foram observadas algumas telhas quebradas (Figuras 5 e 6), com pedaços de telhas posicionados sobre elas e falta de vedação nos pontos de ligação da telha com os parafusos de fixação.

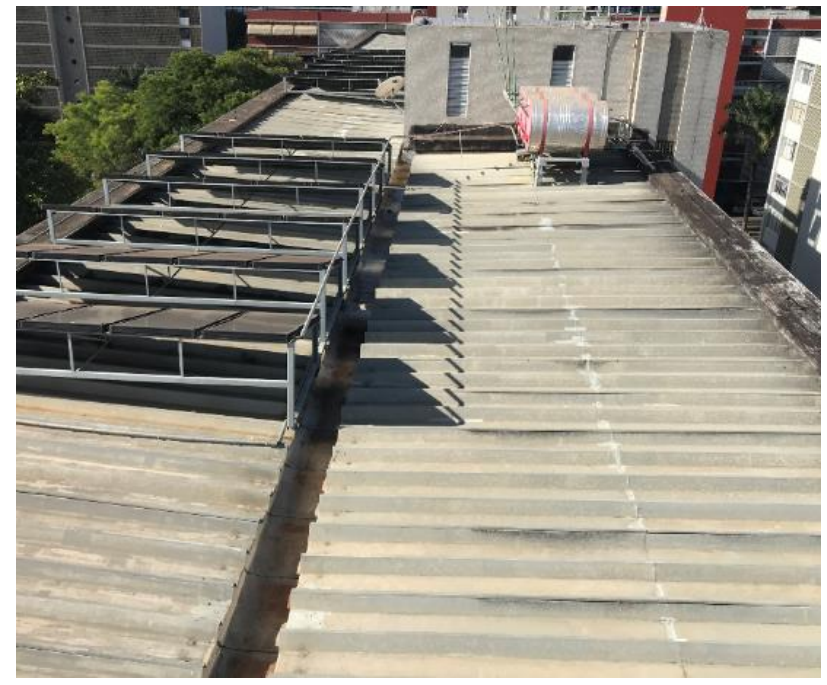

Figura 3: Falta de calafetação das telhas

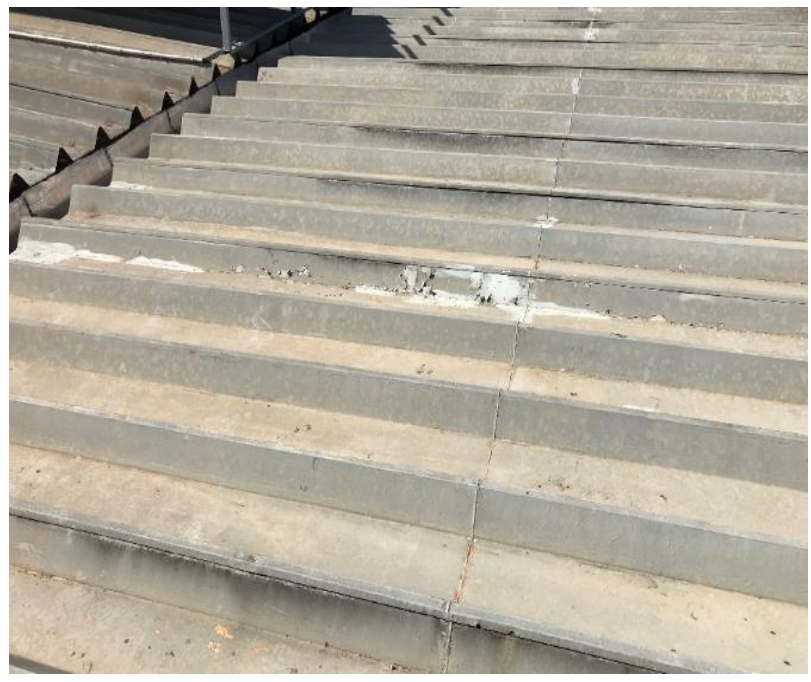

Figura 4: Falta de calafetação e (ou) substituição das telhas

Estes defeitos na vedação podem ocasionar infiltrações, que provocam uma cadeia de eventos que contribuem para a degradação das estruturas de concreto armado favorecendo a corrosão das armaduras e consequente destacamento do concreto que o recobre. Além disso, ambientes internos dos apartamentos do pavimento superior podem perder condições de salubridade e habitabilidade por umidade excessiva, com geração de fungos que podem provocar problemas respiratórios.

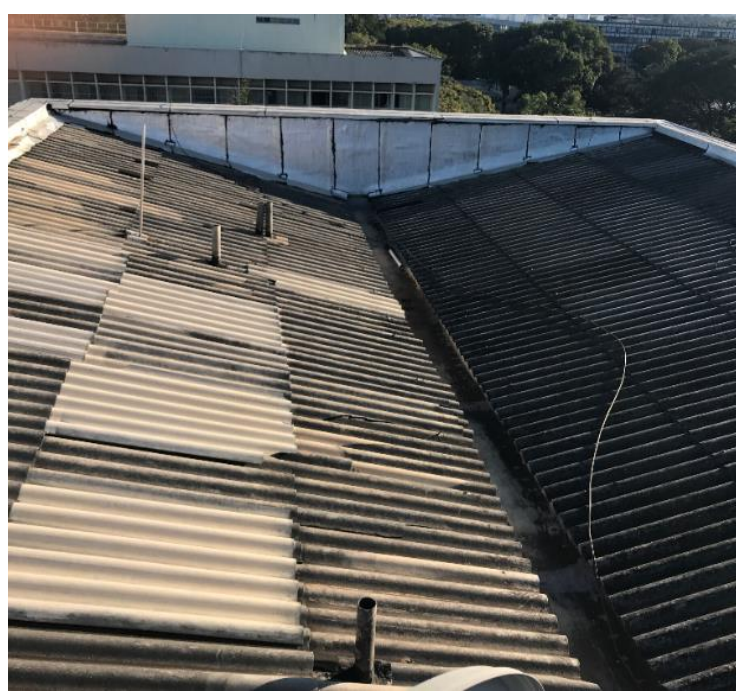

Figura 5: Telhas quebradas

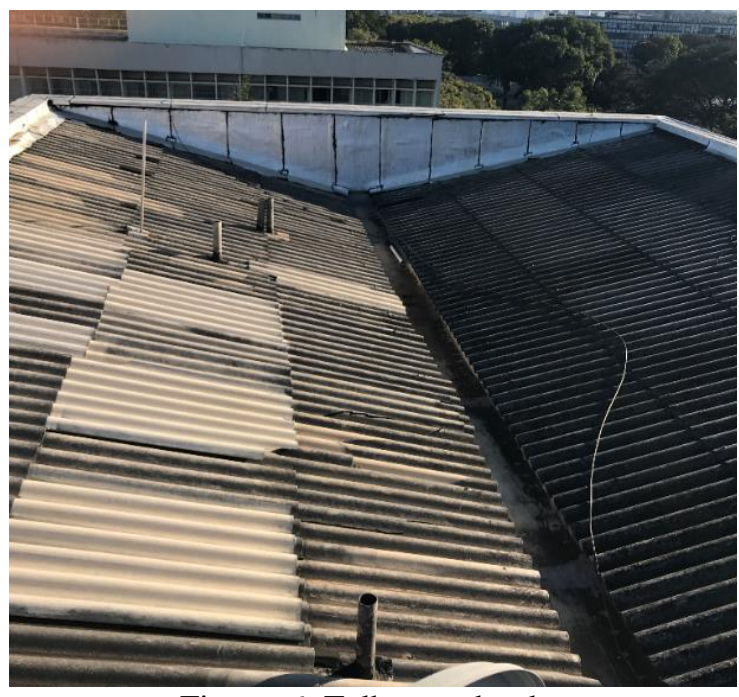

Figura 6: Telhas quebradas 
Classificação do Problema: Falha na manutenção;

Causas: Houve desgaste natural ao longo do tempo e falta das devidas manutenções periódicas indicadas pelo fabricante.

Intervenção: Recomenda-se substituir as telhas que apresentam rachaduras, que estejam quebradas e (ou) que estejam recobertas por pedaços de telhas. Proceder com a calafetação das aberturas e cabeças de parafusos de fixação da telha para uma perfeita vedação dessa abertura.

\section{Risco: M (Grau Mínimo)}

\subsection{Falta de terminais nas colunas de ventilação do esgoto}

Os terminais de ventilação do esgoto servem para emitir para a atmosfera os gases e, também, permitir a entrada de ar no interior das tubulações de esgoto, aliviando a pressão que os gases produzidos nessas instalações exercem sobre os fechos hídricos dos aparelhos sanitários.

Manifestações patológicas: Falta de conexão de PVC do tipo Terminal de Ventilação no topo do tubo para as colunas de ventilação do esgoto, como é possível observar nas Figuras 7 e 8.

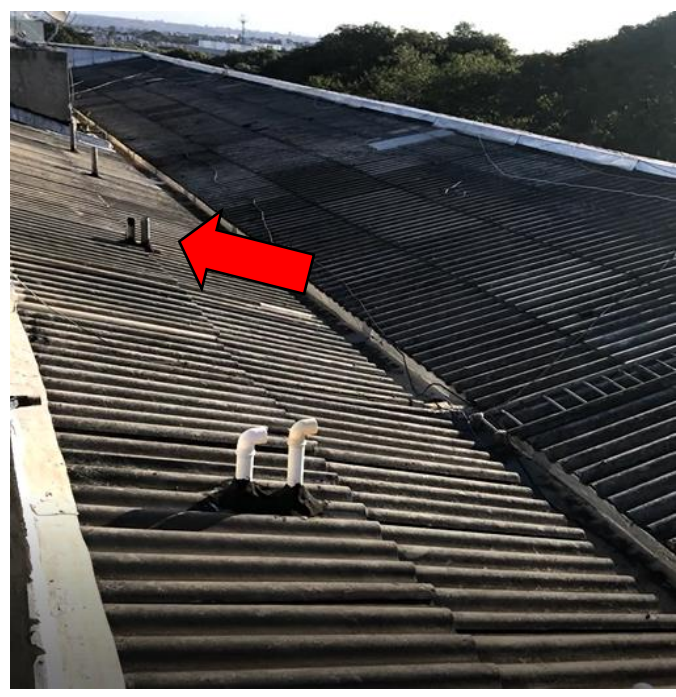

Figura 7: Falta de terminais de ventilação

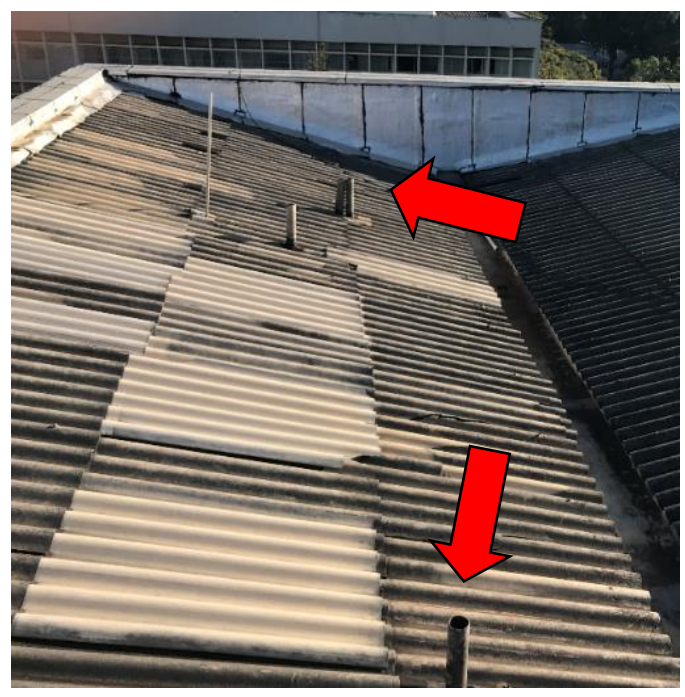

Figura 8: Falta de terminais de ventilação

Classificação do Problema: anomalia construtiva;

Causas: Falta de utilização de uma conexão tipo terminal de ventilação (ou joelho de 90 graus, menos recomendado) no topo dos tubos de suspiro. A falta dessa conexão permite o escoamento de águas de chuvas para dentro dos tubos que deveriam servir somente para eliminação de gases provenientes do esgoto do edifício.

Intervenção: Recomenda-se utilizar o terminal de ventilação (Figura 9), (ou joelho de 90 graus, embora menos recomendado) no topo dos tubos de ventilação e proceder as inspeções periódicas dos tubos ao longo do tempo de utilização;

Risco: M (Grau Mínimo). 


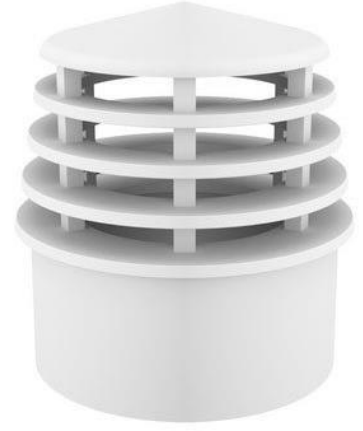

Figura 9: Terminal de ventilação (Fabricante Tigre, 2019).

\subsection{Buzinotes obstruídos}

Os buzinotes fazem parte do sistema de drenagem da edificação, e permitem o escoamento de águas pluviais da cobertura, o entupimento desse sistema, compromete o seu funcionamento, principalmente, em épocas de chuvas mais intensas.

Manifestações patológicas: alguns dos buzinotes (drenos) das lajes de teto dos reservatórios encontram-se entupidos com matéria orgânica e vegetação, além do próprio material impermeabilizante, como é possível observar nas Figuras 10 e 11.

Classificação do Problema: Falha na manutenção e anomalia construtiva;

Causa: Durante a regularização da argamassa sob as mantas asfálticas não foi previsto rebaixo em torno dos drenos, para que quando fossem aplicadas as mantas, os drenos não tivessem seus diâmetros originais reduzidos. Isto causou restrição do diâmetro e, portanto, ao escoamento da água da superfície, com acúmulo de matéria orgânica que, com a umidade, permite o desenvolvimento de plantas no local, o que restringe ainda mais a passagem de água, obstruindo a boca do tubo em um ciclo vicioso. É possível, ainda, que haja pequenas fissuras nas mantas que aumentem o acúmulo de umidade. Isto pode ser melhor verificado com a remoção das plantas e limpeza do local.

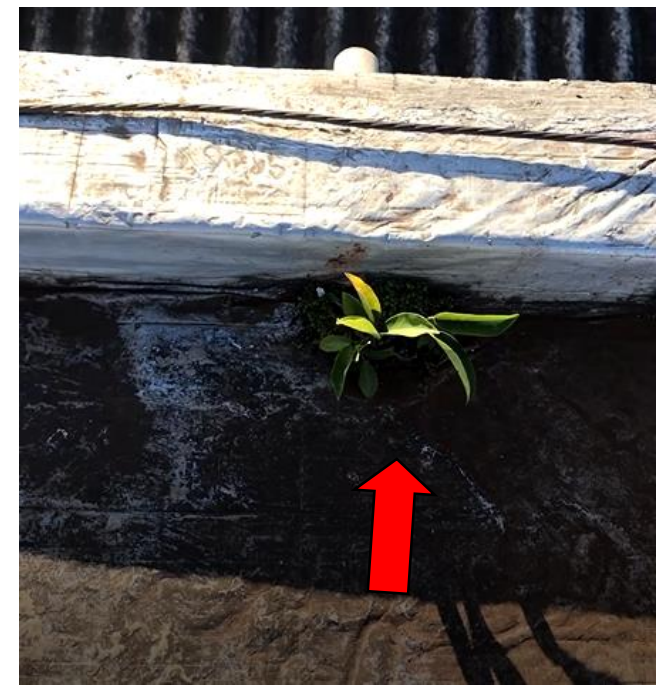

Figura 10: Buzinotes com abertura interrompida

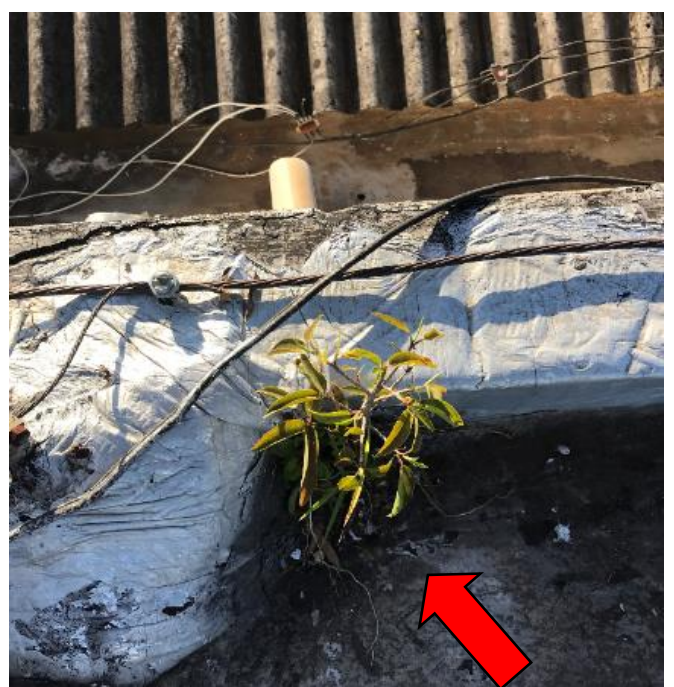

Figura 11: Buzinotes com abertura interrompida

Intervenção: Primeiramente, recomenda-se remover as plantas, mantendo os buzinotes desobstruídos e limpos periodicamente, porém sugere-se a substituição por drenos com diâmetros maiores (mínimo 75 mm) para evitar o entupimento no caso de chuvas mais intensas. Para manter este diâmetro, o sistema de impermeabilização deve ser 
refeito, especialmente neste local, com previsão de rebaixos em torno destes drenos. Com a remoção das plantas deve-se observar se há outros prejuízos à integridade das mantas naqueles locais.

Risco: R (Grau Regular).

\section{CONCLUSÃO}

Dentre os itens inspecionados, os que exigem maior atenção está o desgaste da impermeanilização da cobertura, que pode resultar em infiltrações nos pavimento inferiores, este é um problema que com o tempo pode provocar danos estruturais através da corrosão de armaduras dentro da estrutura de concreto armado.

De uma forma geral, pode-se dizer que os demais problemas surgem em virtude dos edifícios serem antigos, além de terem sofrido diversas intervenções ao longo de sua história, resultando em anomalias construtivas. Muitos de seus elementos construtivos se encontram, portanto, em situações suscetíveis à apresentação de manifestações patológicas, pois estão se deteriorando naturalmente.

As manutenções preditivas são fundamentais para a extensão da vida útil da edificação como um todo, fundamentadas em monitoramentos periódicos ao longo de sua vida útil. As sugestões de intervenções estruturais mencionadas neste trabalho são de caráter corretivo e preventivo, e buscam garantir a segurança dos condôminos e demais usuários.

A fim de manter a qualidade, a segurança e o desempenho das edificações, é necessário a realização de pesquisas voltadas para a área das patologias construtivas, suas causas e soluções, com o objetivo de fornecer instruções e assistência aos profissionais atuantes nessa área da construção civil, para que não apenas o sistema de cobertura, mas todos os sistemas que compõem as edificações, estejam sempre dentro das normas de desempenho e segurança.

\section{AGRADECIMENTOS}

Agradecemos a empresa JR \& Chater Engenharia por disponibilizar e auxiliar na elaboração desta pesquisa.

\section{REFERÊNCIAS}

ASSOCIAÇÃO BRASILEIRA DE NORMAS TÉCNICAS. NBR 9575: Impermeabilização - Seleção e Projeto. Rio de Janeiro, 2010.

ASSOCIAÇÃO BRASILEIRA DE NORMAS TÉCNICAS ABNT: NBR 5674 Manutenção de edificaçõesprocedimento, 1999.

ASSOCIAÇÃO BRASILEIRA DE NORMAS TÉCNICAS. NBR 5674: Manutenção de edificações - Requisitos para o sistema de gestão de manutenção. Rio de Janeiro, 2012.

Instituto Brasileiro de Avaliações e Perícias de Engenharia de São Paulo. Norma de Inspeção Predial. São Paulo, 2011. Disponível em: <http://www.ibape-sp.org.br/arquivos/norma_de_inspecao_predial.pdf>. Acesso em: 13 dezembro de 2019.

GOMIDE, T. L. F.; NETO, J. C. P.; GULLO, M. A. Normas Técnicas para Engenharia Diagnóstica em Edificações. São Paulo: Pini, 2009.

SOUZA, V. C.; RIPPER, T. Patologia, Recuperação e Reforço de Estruturas de Concreto - Pini - São Paulo - SP, 2009; 